\title{
A New Torsion Wave Propagation Model Inversion Algorithm of Pile Foundation Based on Hybrid Artificial Bee Colony Algorithm
}

\author{
Kang Weixin, Ye Youdao* and Zhao Tianwei \\ College of Information and Communication Engineering, Harbin Engineering \\ University, Harbin, 150001, China \\ kangweixin@hrbeu.edu.cn*yeyoudao@hotmail.com zhaotianwei@hrbeu.edu.cn
}

\begin{abstract}
In pile foundation defects detection field, how to modeling the torsion wave propagation in pile foundation to describe the correct relationship of pile and soil is an very important subject, because it will involve the post processing of the engineering data, especially in data fitting accuracy and quantitative analysis of defects. Meanwhile, how to obtain accurate parameters of pile such as pile length, the defect location, the changes of wave impedance by inversion algorithm for qualitative and quantitative analysis of characteristics of the defect is also a difficult problem. The traditional inversion algorithm such as the simplex method has poor convergence because of its searching rule and ending condition, and its inversion accuracy is not ideal.

In this paper, firstly, considering the effect of soil, the torsion wave propagation model is deduced and obtained. Then by introducing an improved hybrid artificial bee colony algorithm, combined with the finite difference algorithm to solve torsion wave propagation model, a new inversion algorithm for torsion wave propagation model is put forward to solve these two problems. Three groups experiment have been done to get these inversion parameters and analyze the defect information in pile. Simulation shows that, relative to traditional inversion algorithm, the inversion algorithm has high precision, stable convergence, and the measured curve fitting well. It can provide accurate parameters for quantitative analysis of defect in pile foundation.
\end{abstract}

Keywords: Artificial bee Colony Algorithm; Torsion wave propagation model; Pile foundation; inversion analysis; Finite difference algorithm

\section{Introduction}

Relative to longitudinal wave, the torsion wave propagation model has significant advantages in the interface reflection of pile body. The attenuation rule of torsion wave response contains a lot of defect information and pile parameters information, and if the torsion wave propagation can be built up and these information can be obtained by inversion algorithm, then a qualitative and quantitative analysis of pile defects can be much clearly and precisely analyzed.

Cai Zheng[1] developed an analytical solution in his paper to investigate the dynamic response of a large-diameter end-bearing pipe pile subjected to torsion loading in viscoelastic saturated soil. And the wave propagation in saturated soil and pile was simulated by Biot's two-phased linear theory and one-dimensional elastic theory, respectively. Wenbing $\mathrm{Wu}[2]$ investigated the dynamic torsion response of a pile embedded in layered soil while considering the influence of the pile and soil. And then he obtained the analytical solution for the dynamic response of the pile head in the frequency domain and derived the relevant semi-analytical solution in the time domain by using the Fourier transform and convolution theorem. And Yuanqiang Cai[3] used an analytical approach to study the torsion vibrations of a rigid circular foundation resting on saturated soil to obliquely incident SH waves. Numerical results were presented demonstrate the 
influence of excitation frequency, incident angle, the torsion inertia moment of the foundation and permeability of the saturated half-space on the torsion vibrations of the foundation.

Sudip Basack[4] presented a novel numerical model (boundary element method) to analyze the response of a single, vertical, floating pile subjected to pure torsion load. The effect of progressive pile-soil slippage at the interface was considered, too. And the proposed model was also applied successfully to selected case studies on concrete piles ( $L / D$ ranged from 10-40) in medium-soft clay, medium-dense sand, and layered soil, and a set of design curves had been constructed. Xuanming Ding[5] presented an analytical solution used to investigate the wave propagation in a pipe pile under an axial point load and applied in case studies. Wang Jinhai[6] applied inversion of 3-parameter theory to accurately match the deflection, rotation, moment, shear tested on the top of laterallyloaded pile, and the inversion method was given in his paper. JIANG Kaile[7] given a method of relatively accurate numerical of soil resistance factor using related theory and field monitoring information. And the soil resistance factor was inverted by combining the backfill soil monitoring information with $\mathrm{m}$ method in the engineering foundation. Jiangong Chen[8] applied the genetic algorithm to inverse parameters of integrated pile, the specific procedure was also given in his paper.

In this paper, firstly, the mathematical model of torsion wave propagation in pile foundation is deduced, and by combining hybrid artificial bee colony algorithm and finite difference algorithm, a new inversion analysis search algorithm is proposed, three groups experiment proved that this algorithm can inverse the length of pile, wave velocity, defect radius and its location, density of pile and so on much accurately, so does generalized wave impedance.

\section{Mathematical Model of Torsion Wave Propagation in pile foundation}

The classical stress wave reflection method is given priority to longitudinal wave, and widely applied to pile dynamic tests. While there exists blind detect area and not sensitive to deep small defects when using longitudinal wave to detect the defects; The torsion wave hold sensitive characteristics of shallow and deep small defects make up for the deficiency of the longitudinal wave, therefore, in this paper, the main work is to combine the existing torsion wave propagation theory and related research achievements of pile soil system, considering the interaction of pile and soil, then build up an mathematical model of torsion wave propagation in pile foundation.

Assuming that pile soil is horizontal layer, each layer are uniform and isotropic, and linear elastic constitutive model assumption of pile body; Pile subsoil is homogeneous isotropic elastic half space; Pile is an upright, linear elastic piecewise uniform circular rod; Without separation between pile surface and soil (see Figure 1). Pile and soil are divided into $n$ connected segments according to different defect type. The parameters of segment $j$ are: the density $\rho_{\mathrm{j}}$, shear modulus $G_{j}$, radius of $r_{j}$, length $l_{j}=h_{j}-h_{j-1}$; The corresponding parameters of pile soil are: density $\rho_{\mathrm{sj}}$, shear wave velocity ${ }_{s j}$; Pile soil parameters: density $\rho_{\mathrm{b}}$, shear wave velocity ${ }_{s b}$. 


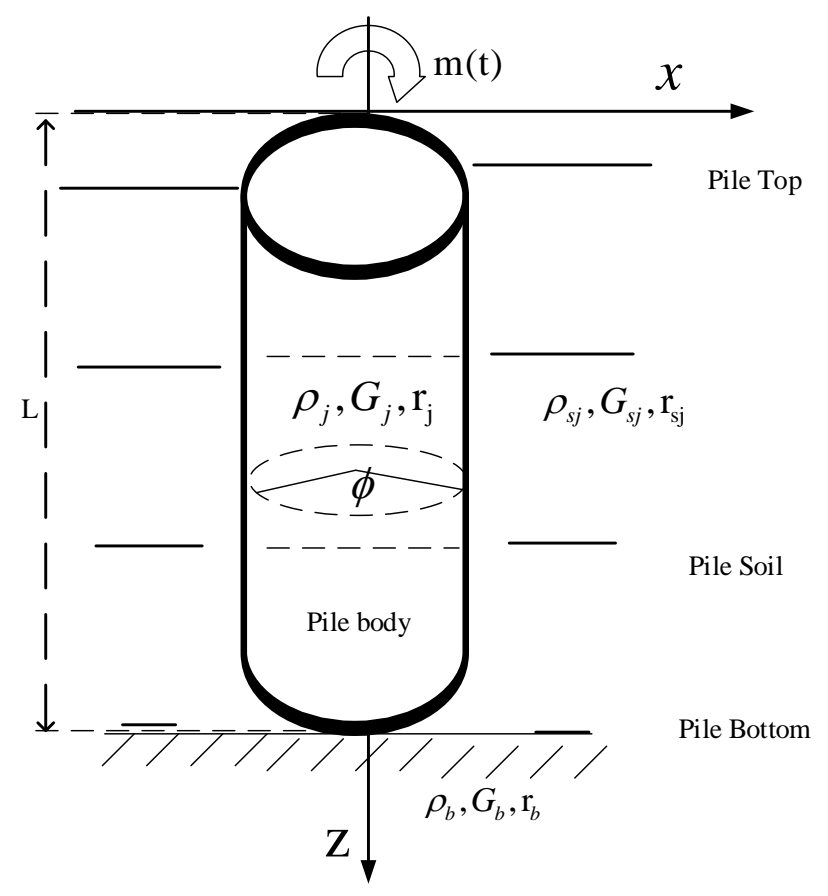

Figure 1. Torsional Wave in the Pile Foundation Modal

At the top of pile affect an excitation torque $m(t)$ to make pile for transient torsion vibration. Torsion vibration angle $\theta_{j}(z, t)$ satisfy the differential equation:

$G_{j} J_{j} \frac{\partial^{2} \theta_{j}(z, t)}{\partial z^{2}}+m_{s j}=\rho_{\mathrm{j}} J_{j} \frac{\partial^{2} \theta_{j}(z, t)}{\partial t^{2}}, j=1,2, \ldots, n$

In equation(1), ${ }^{m}$ ij is excitation torque effected by soil torque to segment $j$ of pile; ${ }^{J}{ }_{j}$ is polar moment of inertia of segment $j$ cross section and $J_{j}=\pi r_{j}^{2} / 2$.

Initial conditions as follows:

$\left.\theta_{j}(z, t)\right|_{t=0}=0,\left.\frac{\partial \theta_{j}(z, t)}{\partial t}\right|_{t=0}=0, j=1,2, \ldots, n$.

Interface joins condition for adjacent pile segment determined by the continuity of torsion angle and torque,

$$
\begin{aligned}
& \text { namely }\left.\theta_{j}(z, t)\right|_{z=h_{j}}=\left.\theta_{j+1}(z, t)\right|_{z=h_{j}}, \\
& \left.G_{j} J \frac{\partial \theta_{j}(z, t)}{\partial z}\right|_{z=h_{j}}=\left.G_{j+1} J_{j+1} \frac{\partial \theta_{j+1}(z, t)}{\partial z}\right|_{z=h_{j}}, j=1,2, \ldots, n-1 .
\end{aligned}
$$

Pile top boundary condition is as follows:

$\left.G_{1} J_{1} \frac{\partial \theta_{1}(z, t)}{\partial z}\right|_{z=0}=-m(t)$

Pile bottom boundary condition is as follows: 


$$
\left.G_{n} J_{n} \frac{\partial \theta_{n}(z, t)}{\partial z}\right|_{z=h_{n}}=-m_{b}
$$

$m_{b}$ is the torque from pile subsoil to pile bottom,

Stiffness coefficient and damping coefficient of pile soil[9] is:

$$
\left.\begin{array}{c}
k=3 \pi \rho_{s} v_{s}^{2} \\
c=2 \pi r_{0} \rho_{s} v_{s}^{2}
\end{array}\right\}
$$

Stiffness coefficient of pile subsoil[10]:

$$
k_{b}=(32 / 9) \rho_{b} v_{s b}^{2} r_{n}^{3}
$$

Damping coefficient of pile subsoil:

$$
c_{b}=(\pi / 2) \rho_{b} v_{s b} r_{n}^{4}
$$

\section{Inversion Algorithm for Torsion Wave Propagation in Pile Foundation}

The mathematical model of torsion wave propagation given above can be solved by finite difference algorithm.

So, next, based on artificial bee colony algorithm[11], an improved hybrid artificial bee colony is proposed and combined with the finite difference algorithm[12] to get numeric solution of velocity response $V^{\prime}(t)$ according to the mathematical model of torsion wave propagation, then the torsion wave propagation model inversion algorithm is designed.

This inversion algorithm has stronger robust performance and faster convergence speed and higher convergence precision and it's helpful to improve the calculation accuracy and convergence problems of traditional inversion algorithm.

The main idea of inversion algorithm is shown as Figure 2. Figure 2 show the flow chart of the inversion search algorithm.

From Figure 2 can we see that, by a bell-shaped pulse excitation $m(t)$, the measured response curve of wave propagation in model pile $V(t)$ is obtained, then $V(t) i s$ as an input of inversion search algorithm. The search solution generated by hybrid artificial bee colony algorithm input to mathematical model of torsion wave propagation to get the inversion velocity response $V^{\prime}(t)$. The measured velocity response curve $V(t)$ and the inversion velocity response $V^{\prime}(t)$ plug in the objective function to get the fitness value. And according to the fitness value to adjust hybrid artificial bee colony algorithm search direction, until exceed a specified number of iterations or achieve specified accuracy, then output inversion results. The results are these factor which will effect torsion wave spread in pile foundation, such as pile length $L$; pile radius $R$; density $\rho^{\rho}$; wave velocity $C_{t}$; stiffness coefficient $k$ and damping coefficient $c$ for soil; stiffness coefficient $k_{b}$ and damping coefficient $c_{b}$ for pile subsoil . 


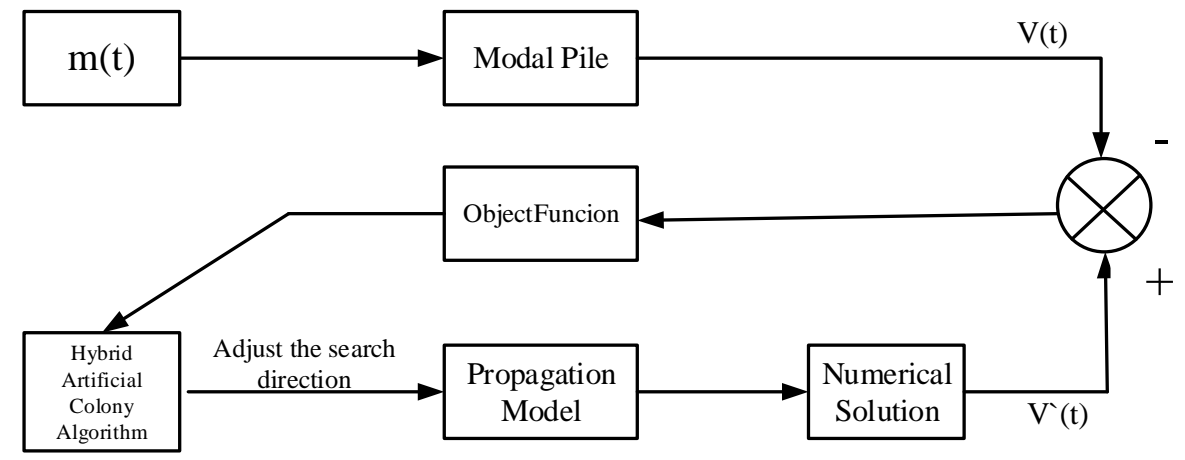

Figure 2. Inversion Algorithm Block Diagram

And the steps of inversion algorithm of torsion wave propagation model are as follows.

Step 1: parameters setting: bee number $N p$, foods source number $n e=N P / 2$, local search limitation number limit, maximum cycle $G$, dimension to be changed $M D$, the number of bacterial chemotaxis operation $N s$, initial pressure value $T_{0}$, pressure control $\alpha$, chaos iterations $N_{\max }$. D for dimension of solution vector [Lmatrix,Rmatrix,Pmatrix,Em,Uu,Vsmatrix,Psmatrix,Vsb,Psb,Ub].

Read measured transient response at pile top, mainly measured velocity response $V(t)$ as an input to inversion algorithm.

Step 2: random solution $X_{i}(i=1,2, \ldots, N P / 2)$, using equation(13) to generate new solution $V_{i}$ of $N p / 2$ employed bees, $\Phi_{i j}$ is a random number between $[-1,1]$, and initialize the counter failure $_{i}=0$; This vector including almost all parameters that determined torsion wave propagation modal and will be output at end.

Input solutions to torsion wave propagation and get numeric solution of velocity response $V^{\prime}(t)$ according to reference [8]. $V^{\prime}(t)$ is the inversion velocity response ,the main steps to calculate $V^{\prime}(t)$ value with solutions are:

$v_{n}^{1}=0, \quad v_{n}^{2}=0 ; \quad n=1,2, \ldots, N$

$v_{1}^{m}=v_{2}^{m}+\left.\frac{\Delta z}{T(1)} r_{\mathrm{t}} \frac{d m(t)}{d t}\right|_{t=(m-1) \Delta t}$

$v_{N}^{m+1}=\mathrm{B} v_{N-1}^{m+1}+D v_{N}^{m}$

Whereas:

$B=\frac{1}{q_{3}}, \quad D=\frac{q_{1}}{q_{3}}, \quad q_{3}=1+q_{1}+q_{2}, \quad q_{1}=\frac{c_{b} \Delta z}{T(N) \Delta t}, \quad q_{2}=\frac{k_{b} \Delta z}{T(N)}$

The objective function is:

$f\left(V^{\prime}(t)\right)=\left.\frac{\operatorname{sum}\left(\left(V(t)-V^{\prime}(t)\right)^{2}\right)}{N}\right|_{V_{i}}$

Where $V^{\prime}(t)$ is the inversion velocity response, $N$ is discrete points of time. Actually, $f$ is then mean squared error function and it also can be used as fitting precision.

Calculate the fitness value by equation(14) and the Boltzmann probability $P_{i}$ by equation(15) for corresponding bee $i$.

$V_{i j}=X_{i j}+\Phi_{i j}\left(X_{i j}-X_{k j}\right)$ 


$$
\begin{aligned}
& \text { fit }_{i}=\left\{\begin{array}{c}
1 /\left(1+f\left(X_{i}\right)\right), f\left(X_{i}\right) \geq 0 \\
1+\operatorname{abs}\left(f\left(X_{i}\right)\right), f\left(X_{i}\right)<0
\end{array}\right. \\
& P_{i}=\frac{\exp \left(\text { fit }_{i} / T\right)}{\sum_{j=1}^{S N} \exp \left(\text { fit }_{j} / T\right)}
\end{aligned}
$$

Where $f\left(X_{i}\right)$ is the objective function of solution $X_{i}$, calculate $V^{\prime}(t)$ using solution $X_{i}$, then can we get $f\left(X_{i}\right)$. fit $t_{i}$ is the fitness function of food source $i, T$ is a parameter to control selection pressure. When $T$ value becomes bigger, selection pressure decreases accordingly, so parameter $T$ becomes the key to adjust the selection pressure. In order to choose $T$ value dynamically, the equation (16) is used as follows:

$T=T_{0} * \alpha^{g-1}$

Where $T_{0}$ is constant; when $g=1, T=T_{0}$, it determines the initial selection pressure; $g$ for iterations number; $\alpha$ is a parameters of the adaptive speed control, if $\alpha<1, T$ value go with the increase of $g$ exponentially decreases, and thus make selection pressure gradually increase. In order to avoid growing too fast, generally the initial value can be $\mathrm{T}_{0}=100, \alpha=0.995$.

Record the minimum fitness value as the optimal value.

Step 3: For each employed bee, produce new solution by using equation(17) and calculate its fitness value $f_{i t}{ }_{i}$. The main idea of equation (17) is from bacterial foraging $[13,14]$.

$V_{i}=X_{i}+\Delta_{i}$

$$
\Delta_{i}=\bigcup_{j d \dot{o} J, d=1}^{M D}\left[\operatorname{rand}(-1,1) *\left(x_{j d}^{j}-x_{j d}^{k}\right)\right]
$$

Where $M D$ is a positive integer no greater than dimension $D$, representing the bee $i$ can change $M D$ dimension at one time, $M D$ value option for $M D=0.3 * D$. When $M D$ is too small, then the adjustment of fitness value ability is limited, $\left[j_{1}, \ldots, j_{M D}\right]$ is a set of $M D$ numbers $\mathrm{J}, j_{d} \in \mathrm{J}$ is random dimension range from $[1, D]$, and not repeat to each other; $k \in[1,2, \ldots, n e]$ and must meet $k \neq i$.

Calculate the fitness value fit $_{i}$ ' by equation(14) and their Boltzmann probability $P_{i}$ by equation(15) for all employed bees.

If fit $_{i}{ }^{\prime}<$ fit $_{i}$, failure $_{i}=$ failure $_{i}+1$, move to the next step, otherwise make failure ${ }_{i}=0$ and memorize the new position $X_{i}=V_{i}$; Record the minimum fitness value as optimal value.

Repeat the same chemotaxis action, if the bee does not improve the food source more than $N$ s times, move to step 5.

Step 4: for each scout bee, according to the Boltzmann probability $P_{i}$ to choose a food source and then exploit the selected food source as the employed bee does in Step 3.

Step 5: Check all employed bees. If failure ${ }_{i}>$ limit, began to chaos search[15] and the current local optimal solution stored in tabu list. Produce $N_{\max }$ consecutive iterations according to the equation(18):

$$
x_{(n+1), d}=\mu x_{n, d}\left(1-x_{n, d}\right)
$$

Where $n \in\left[1, N_{\max }\right], d \in[1, D] .{ }^{\mu}$ is the control parameter of chaotic state. 
Logistic equation were fully entered into a state of chaos when ${ }^{\mu}=4$.

And then got $N_{\max }$ solutions by equation(19):

$$
x_{n, d}^{\prime}=f_{i, d}+R_{i, d}\left(2 x_{n, d}-1\right)
$$

When the employed bee turned into scout bee, Initial its position by random vector $x_{0}=\left[y_{0,1}, y_{0,2}, \ldots\right]_{D}$, then the Logistic equation iteration for Nmax times to produce Nmax foods for $x_{n, d}^{\prime}$ to $f_{i, d}$ center, radius area $R_{i, d}$.

By calculating fitness value with these new foods source generated by chaos search to decide whether to update the current food source. $R_{i, d}$ decides the size of region to chaos search. By setting the appropriate value of $R_{i, d}$ can help bees escape from local optimum to look for other optimal solutions. $R_{i, d}=0.3 *$ rang is an appropriate value, where rang is the length of search region.

Then the fitness value of new food source compared these value existed in tabu list. If there exist equal fitness value in tabu list, abandon the current food source, continue to chaos search; If not exist, if the fitness value of the current fitness is greater than the local optimal value, then update the current food source, if less than the local optimal value, continued to chaos search. Check all chaos search solutions, if the food source is not updated, then the employed bee abandons the corresponding food source position, random its position by equation(13).

Step 6: Check current iteration number $g$, if $g$ does not exceed the maximum cycle $G$, and the precision does not meet the requirements, go to step $\mathbf{3}$ to perform the next round of cycle. Otherwise do the next step.

Step 7: output optimal results, exit.

\section{Simulation and Analysis}

Based on the mathematical model of torsion wave propagation and the inversion algorithm proposed above, this section will finish the following simulation experiment: First, establish model pile, model pile including non-defect pile, single defect pile and double defects pile, and non-defect pile including friction pile, rock-socketed pile and free pile; single defect including hole shrinkage, hole enlargement, segregation and mixture of mud, and double defects is the combination of single defect, due to different model pile, can we get measured velocity response for different model pile. Second, through inversion algorithm proposed above to fitting the measured velocity response curve, and output the inversion results of relevant parameters.

Hybrid artificial bee colony algorithm parameters settings: set the bee populations $N p=$ 20; Local iteration limit number limit $=200$; The dimensions of the food source to be changed $M D=6$; Food source dimension $D=10$; Number of bacteria chemotaxis $N s=3$; The pressure of the Boltzmann probability factor $T_{0}=100, a=0.995$; The number of iterations $G=400$; Chaotic iterations $N \max =30$, chaos control parameter ${ }^{\mu}=4$.

Parameters for different kind of pile:

Integrate pile parameters setting: pile length $L=10$; Radius $R=0.3$; Density $\rho=2400$; Elastic modulus $E=2.4576 e 10$; Poisson ratio $\mu=0.28$;

Shear wave velocity of pile soil $V_{s}=50$; The pile soil density $\rho_{\mathrm{s}}=1700$; Shear wave velocity in pile bottom $V_{s b}=50 ; \rho_{\mathrm{sb}}=1700$; poison ratio of pile at bottom $\mu_{b}=0.4$; excitation impulse $\mathrm{I}=1$; excitation time $\mathrm{T}_{\mathrm{m}}=1$.e- 3 ;

Single defect pile: hole shrinkage $R=0.25$; hole enlargement $R=0.4$; the defect length: 
$0.5 \mathrm{~m}$, location $L 1=5 \mathrm{~m}$; Segregation density is 0.6 , mixture of mud density is 0.85 ;

Double defects pile: pile radius $R=0.6$; hole shrinkage $R=0.4$; hole enlargement $R=$ 0.8 ; Defect location $L 1=5 \mathrm{~m} ; L 2=5.5 \mathrm{~m} ; L 3=10 \mathrm{~m} ; L 4=20 \mathrm{~m}$; The discussion and inverse analysis simulation below are given priority to friction pile, the hierarchical model for pile soil and other parameters are the same as above;

The inversion simulation experiments based on model piles, the inversion parameters are: the length of pile Radius $R$, density $\rho$; elastic modulus $E$; poison ratio of pile ${ }^{\mu}$; The pile soil shear wave velocity $V_{s}$; The pile soil density $\rho_{\mathrm{s}}$; pile bottom shear wave velocity $V_{s b}$; The density of pile subsoil $\rho_{\mathrm{sb}}$;Poisson ratio at the bottom of pile;

Then inversion search algorithm will output all of the above parameters after 400 times iterations.

In this paper, these parameters are given: pile length $L$; pile radius $R$; density ${ }^{\rho}$; wave velocity $C_{t}$; stiffness coefficient $k$ and damping coefficient $c$ for soil; stiffness coefficient $k_{b}$ and damping coefficient $c_{b}$ for pile subsoil. Stiffness coefficients and damping coefficient are obtained by using the simplified type. Double defect pile using the average velocity $C_{t}$ ' instead of the velocity of each segment.

In fact, if we are interested in generalized wave impedance $Z$, where $z=\rho C_{t} A R_{g}{ }^{2}$, it can also be calculated by these parameters given above and then be added to tables below.

\subsection{Integrity Pile Inversion Analysis}

Figure 3, Figure 4, and Figure 5 are given the inversion curve of velocity response for friction pile, rock-socketed pile and free pile. Diagram (a) shows the inverse velocity curve and measured velocity curve fitting results; diagram (b) shows the iteration fitting error of both velocity response curves after 400 times iteration.

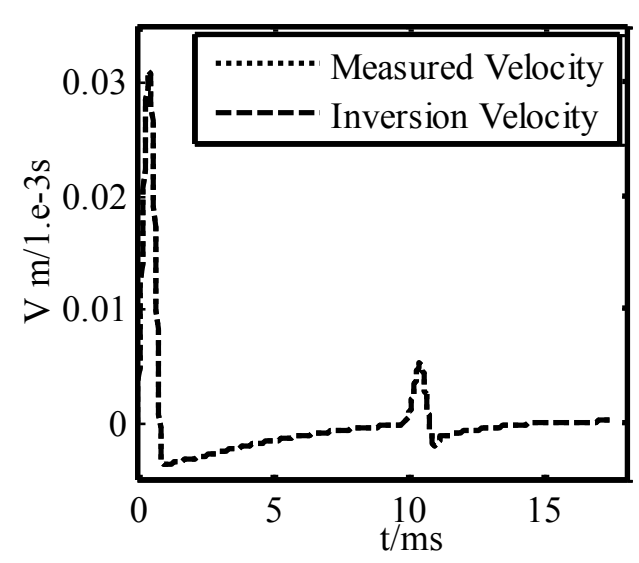

(a)Inversion curve: Velocity - Time

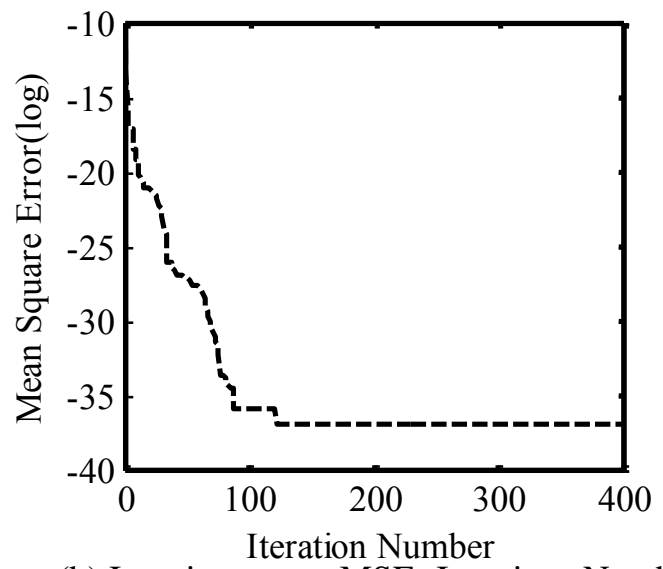

(b) Iterative curve: MSE -Iterations Number

Figure 3. Inversion Analysis of Friction Pile, $\mathrm{L}=10$

Table 1. Iteration Results of Friction Pile

\begin{tabular}{c|c|c|c|c}
\hline & $\mathrm{L}$ & $\rho$ & $\mathrm{E}$ & $\mu$ \\
\hline Output & 10.000 & 2399.999 & $2.4576 \mathrm{e}+10$ & 0.250 \\
\hline Real & 10 & 2400 & $2.4576 \mathrm{e}+10$ & 0.28 \\
\hline & $\mathrm{k}$ & $\mathrm{c}$ & $\mathrm{k}_{\mathrm{b}}$ & $\mathrm{c}_{\mathrm{b}}$ \\
\hline Output & 40055544.08 & 160221.100 & 469119.7602 & 1159.674 \\
\hline Real & $4.0055 \mathrm{e} 7$ & $1.6022 \mathrm{e} 5$ & $4.08 \mathrm{e} 5$ & $1.0815 \mathrm{e} 3$ \\
\hline
\end{tabular}


Table 1, Table 2, and Table 3 corresponding to Figure 3, Figure 4, Figure 5 are given the iterative parameters result which will output by the inversion algorithm, real corresponding real parameter values used in model pile and output means the output parameters result of the inversion algorithm in these tables below.

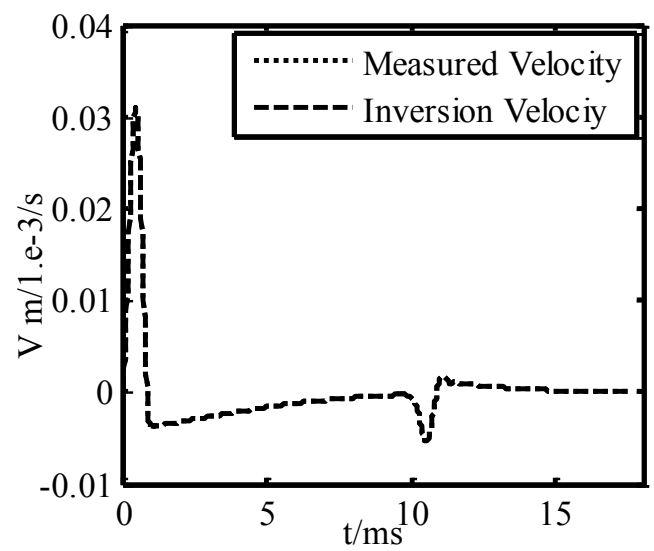

(a) Inversion curve: Velocity - Time

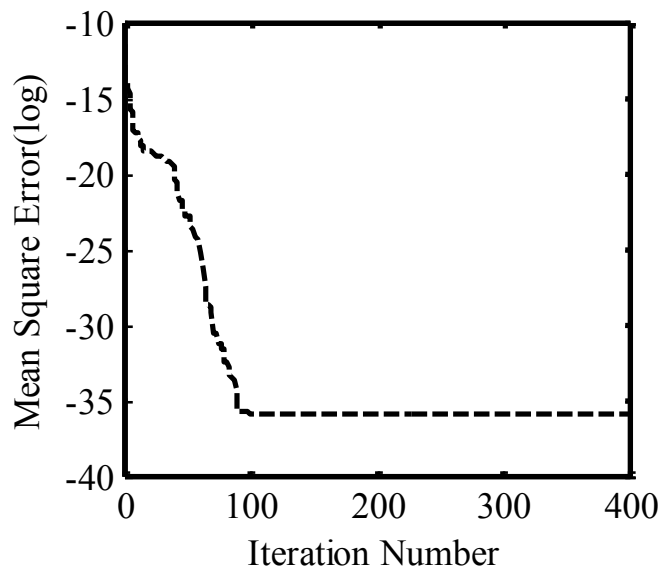

(b)Iterative curve: MSE -Iterations Number

Figure 4. Inversion Analysis of Rock-socketed Pile, L=10

Table 2. Iteration Results for Rock-socketed Pile

\begin{tabular}{c|c|c|c|c}
\hline & $\mathrm{L}$ & $\rho$ & $\mathrm{E}$ & $\mu$ \\
\hline Output & 9.963 & 2399.999 & $2.4617 \mathrm{E}+10$ & 0.278 \\
\hline Real & 10 & 2400 & $2.4576 \mathrm{e}+10$ & 0.28 \\
\hline & $\mathrm{k}$ & $\mathrm{c}$ & $\mathrm{k}_{\mathrm{b}}$ & $\mathrm{c}_{\mathrm{b}}$ \\
\hline Output & 40056031.26 & 160220.584 & 2246401666 & 96059.822 \\
\hline Real & $4.005 \mathrm{e} 7$ & $1.6022 \mathrm{e} 5$ & $2.2464 \mathrm{e} 9$ & $9.9243 \mathrm{e} 4$ \\
\hline
\end{tabular}

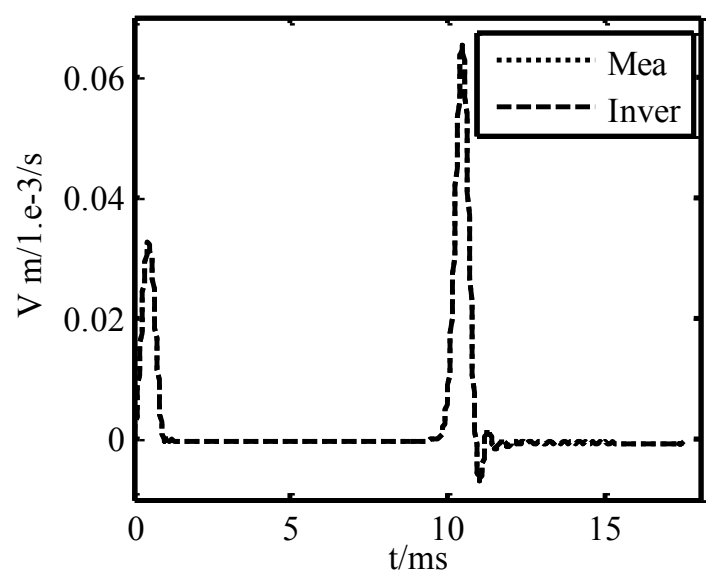

(a)Inversion curve: Velocity - Time

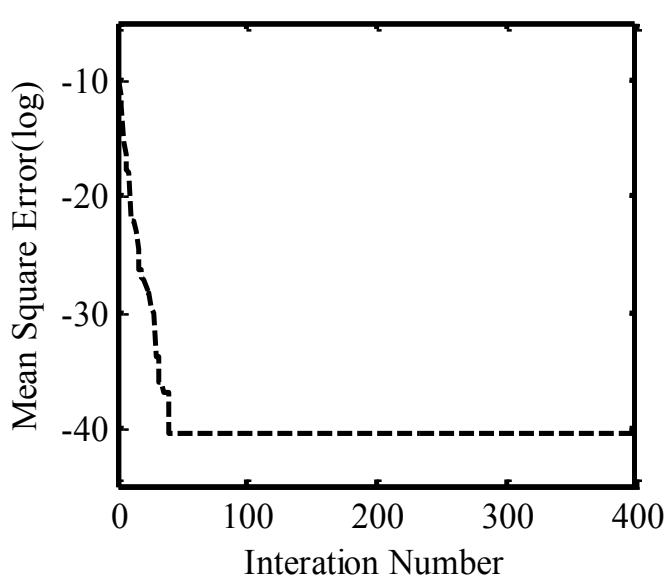

(b) Iterative curve: MSE -Iterations Number

Figure 5. Inversion Analysis of Free Pile, L=10 
Table 3. Iteration Results for Free Pile

\begin{tabular}{c|c|c|c|c}
\hline & $\mathrm{L}$ & $\rho$ & $\mathrm{E}$ & $\mu$ \\
\hline Output & 9.997 & 2400.000 & $2.4576 \mathrm{E}+10$ & 0.260 \\
\hline Real & 10 & 2400 & $2.4576 \mathrm{e}+10$ & 0.28 \\
\hline & $\mathrm{k}$ & $\mathrm{c}$ & $\mathrm{k}_{\mathrm{b}}$ & $\mathrm{c}_{\mathrm{b}}$ \\
\hline Output & 0 & 0 & 0.919 & 0.849 \\
\hline Real & 0 & 0 & 0 & 0 \\
\hline
\end{tabular}

From Figure 3(a) ,Figure 4(a), and Figure 5(a), can we see that the inversion curve can better fit the measured curve, and in Figure 3(b), Figure 4(b), and Figure 5(b) can we see that in the iterations number increase to 100 , the iterative error is less than 1e-35 power, the inversion algorithm can quickly convergence and has satisfied accuracy.

Combined with Table 1, Table2, and Table 3, the output results of parameter iteration can be seen in the three tables. The real parameters of pile are: pile length $L=10$; Radius $R=0.3$; Density $\rho=2400$; Elastic modulus $E=2.4576 e 10$; Poisson ratio ${ }^{\mu}=0.28$; the error between the real parameters and the output parameters of friction pile, rock-socketed pile and free pile can be calculated from the three tables .

Substitute these output parameters into equation (6), equation(7), equation (8), then the stiffness coefficient $k$ and damping coefficient $c$ for soil, stiffness coefficient $k_{b}$ and damping coefficient $c_{b}$ for pile subsoil can be calculated. If needed, the velocity response can also be calculated by these parameters with the relative equation.

So according to these parameters given in the tables, the calculated torsion wave velocity $C_{t}$ are $2023.97,2003.43,2015.48$, the error are $1.2 \%, 0.17 \%, 0.78 \%$ respectively, the error of length is $0 \%, 0.37 \%, 0.03 \%$ respectively. Combined the parameters accuracy and the curve fitting the results, we can conclude that the inversion algorithm can accurately mining the related parameters of torsion wave response curves at the top of pile, and has high fitting precision.

\subsection{Single Defect Pile Inversion Analysis}

Figure 6, Figure 7, Figure 8, Figure 9 were given the inversion curve of velocity response curve for pile under the assumption of hole shrinkage, hole enlargement, segregation and mixture of mud respectively. In each figure, Diagram (a) shows the inversion curve and measured curve fitting results, Diagram (b) shows the iterative fitting error of curve after 400 times iteration Table4, Table 5, Table 6, Table 7 corresponding Figure 6, Figure 7 , Figure 8 Figure 9 are given the output result of parameter iteration after 400 times, the real values in table corresponding parameter values used in model pile. 


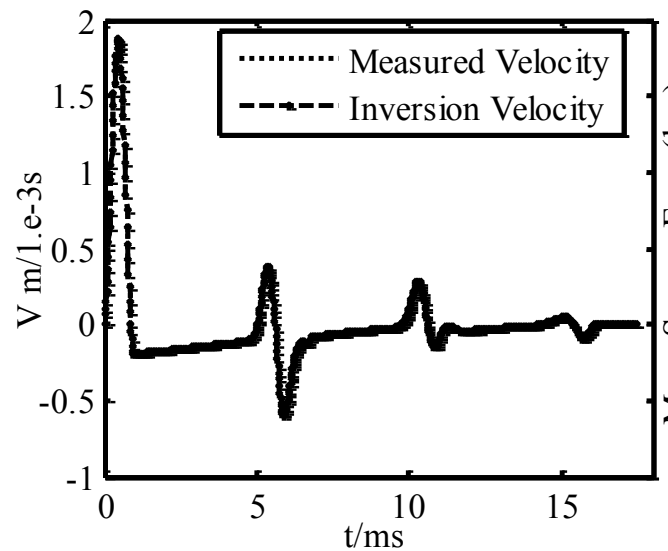

(a) Inversion curve: Velocity - Time

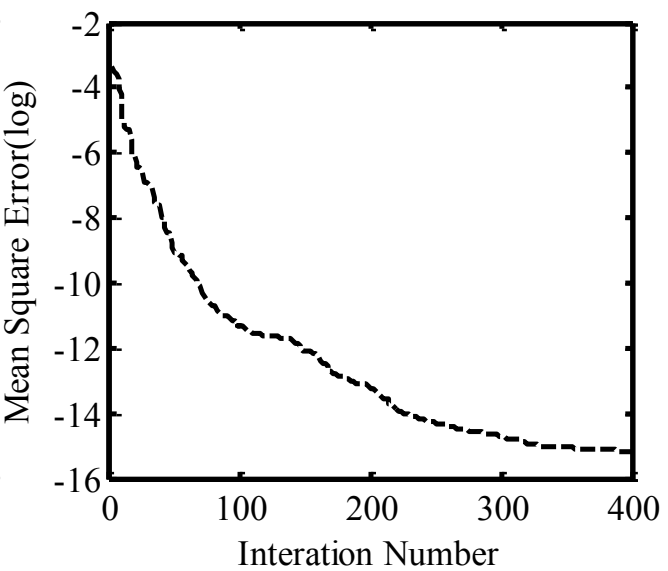

(b) Iterative curve: MSE -Iterations Number

Figure 6. Single Defect- Inversion Analysis for Hole Shrinkage, L1 = 5m; L2 $=5.5 \mathrm{~m} ; \mathrm{L} 3=10 \mathrm{~m}$

Table 4. Single Defect Iteration Results for Hole Shrinkage

\begin{tabular}{c|c|c|c|c|c|c|c|c|c}
\hline & \multicolumn{3}{|c|}{$\mathrm{L}$} & \multicolumn{3}{c|}{$\mathrm{R}$} & \multicolumn{3}{c}{$\rho$} \\
\hline Output & 5.003 & 5.498 & 9.999 & 0.300 & 0.250 & 0.301 & 2398.801 & 2388.252 & 2399.555 \\
\hline Real & 5.0 & 5.5 & 10 & 0.3 & 0.25 & 0.3 & 2400 & 2400 & 2400 \\
\hline
\end{tabular}

The real parameters are: pile radius $R=0.6$; hole shrinkage $R=0.4$; hole enlargement $R=0.8$; the defect length: $0.5 \mathrm{~m}$, location $L 1=5 \mathrm{~m}$; Segregation density is $0.6^{*} \rho$, mixture of mud density is $0.85^{*} \rho$, the double defects share the same parameters.

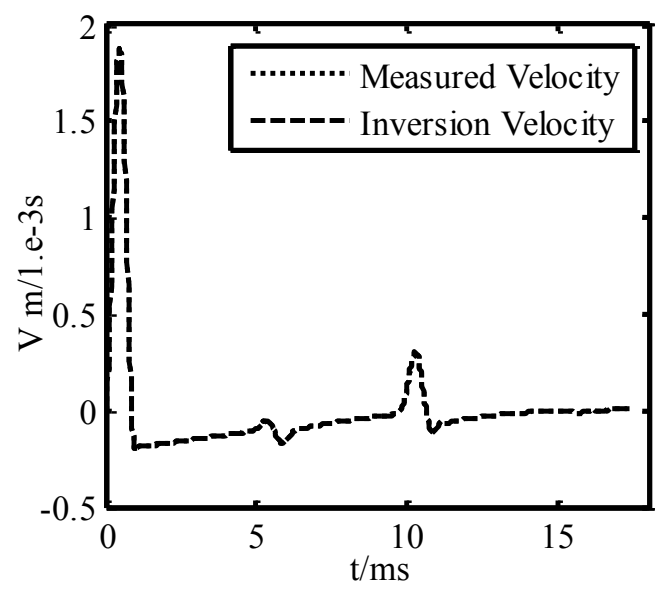

(a)Inversion curve: Velocity - Time

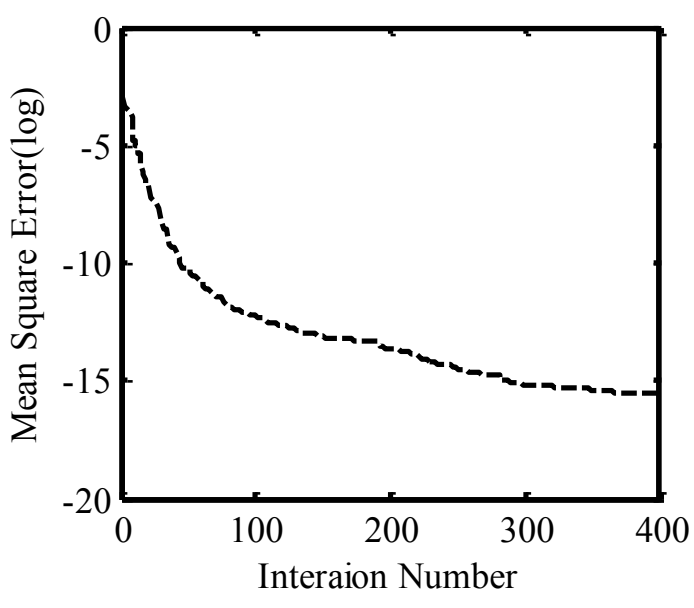

(b)Iterative curve: MSE -Iterations Number

Figure 7. Single Defect - Segregation Defects Analysis, L1 = 5m; L2 = 5.5m; L3 $=10 \mathrm{~m}$

Table 5. Single Defect Iterative Result for Segregation

\begin{tabular}{c|c|c|c|c|c|c|c|c|c}
\hline & \multicolumn{3}{|c|}{$\mathrm{L}$} & \multicolumn{3}{c|}{$\mathrm{R}$} & \multicolumn{3}{c}{$\rho$} \\
\hline Output & 5.004 & 5.500 & 9.989 & 0.300 & 0.301 & 0.299 & 2406.345 & 1958.424 & 2401.079 \\
\hline Real & 5.0 & 5.5 & 10 & 0.3 & 0.3 & 0.3 & 2400 & 1400 & 2400 \\
\hline
\end{tabular}




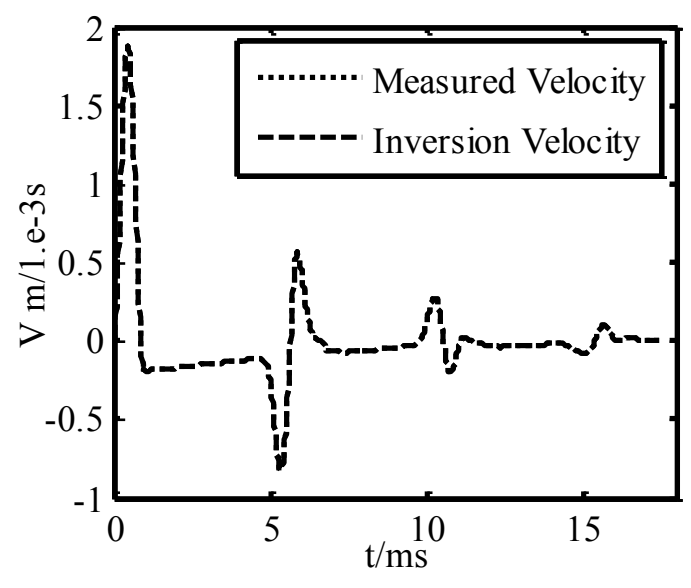

(a)Inversion curve: Velocity - Time

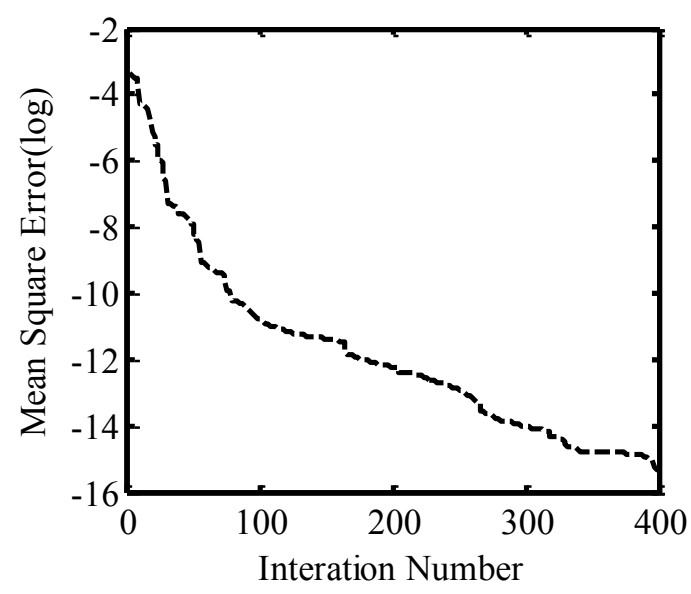

(b)Iterative curve: MSE -Iterations Number

Figure 8. Single Defect - Hole Enlargement, L1 = 5m; L2 = 5.5m; L3 =10m

Table 6. Single Defect Iteration Results for Hole Enlargement

\begin{tabular}{c|c|c|c|c|c|c|c|c|c}
\hline & \multicolumn{3}{|c|}{$\mathrm{L}$} & \multicolumn{3}{c|}{$\mathrm{R}$} & \multicolumn{3}{c}{$\rho$} \\
\hline Output & 5.017 & 5.498 & 9.990 & 0.300 & 0.400 & 0.298 & 2399.852 & 2393.394 & 2401.056 \\
\hline Real & 5 & 5.5 & 10 & 0.3 & 0.4 & 0.3 & 2400 & 2400 & 2400 \\
\hline
\end{tabular}

From Figure 6(a), Figure 7(a), Figure 8(a), Figure 9(a) can we see that the inversion curve can better fit the measured curve, and from Figure 6(b), Figure 7(b), Figure 8(b), Figure 9(b) can be seen that in iterative search algorithm to 400, the iterative error is less than $1 \mathrm{e}-16$ power, the inversion algorithm can quickly stable convergence and has a high accuracy as it can be seen in Diagram (b).

$\mathrm{L}, \mathrm{R}, \rho$ are vector in the tables and, means that $L=\left[\begin{array}{llll}L 1 & L 2 & L 3\end{array}\right], R=\left[\begin{array}{lll}R 1 & R 2 & R 3\end{array}\right]$,

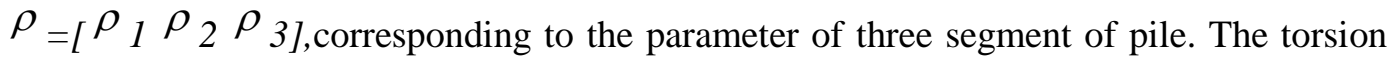
wave velocity will be much complicate and the average velocity can be used to do the error calculating instead.

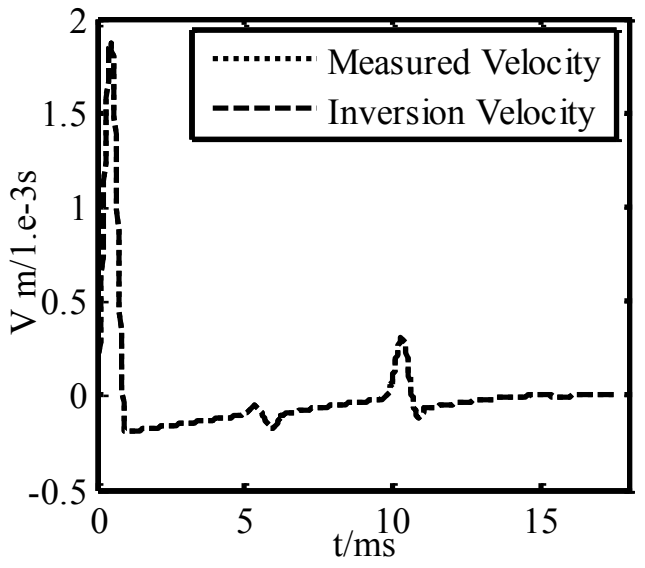

(a)Inversion curve: Velocity - Time

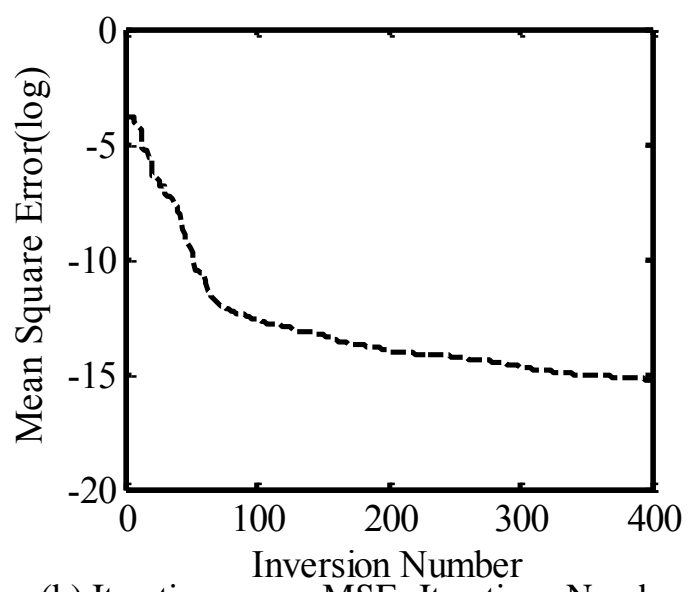

(b) Iterative curve: MSE -Iterations Number

Figure 9. Single Defect -Inversion Analysis for Mix of Mud, L1 = 5m; L2 = $5.5 \mathrm{~m} ; \mathrm{L} 3=10 \mathrm{~m}$ 
Table 7 Single Defect Iteration Results for Mix of Mud

\begin{tabular}{c|c|c|c|c|c|c|c|c|c}
\hline & \multicolumn{3}{|c|}{$\mathrm{L}$} & \multicolumn{3}{c|}{$\mathrm{R}$} & \multicolumn{3}{c}{$\rho$} \\
\hline Output & 4.997 & 5.495 & 9.999 & 0.300 & 0.299 & 0.300 & 2398.039 & 2063.673 & 2396.400 \\
\hline Real & 5.0 & 5.5 & 10.0 & 0.3 & 0.3 & 0.3 & 2400 & 2040 & 2400 \\
\hline
\end{tabular}

The error of average torsion velocity $C_{t}$ ' are $0.32 \%, 0.1 \%, 0.14 \%, 0.26 \%$ respectively. Combined with Table4,Table 5, Table 6 , Table 7 and the output results of parameter iteration can be seen that the inversion algorithm can accurately mining the related parameters of pile body in torsion wave response curves, and has high fitting precision and stable performance.

\subsection{Double Defects Pile Inversion Analysis}

Figure 10, Figure 11, Figure 12, and Figure 13 were given the inversion curve of velocity response curve for pile under the assumption of combination of hole shrinkage, hole enlargement, segregation and mixture of mud respectively. Diagram (a) shows the inverse curve and measured curve fitting results, Diagram (b) shows the iterative fitting error of curve after 400 times iteration. Table 8, Table 9, Table 10, as well as Table11 are corresponding Figure 10, Figure 11, Figure 12, and Figure 13 are given the output result of parameters by the inversion algorithm, the real values in table corresponding parameter values used in model pile.

From Figure 10(a), Figure 11(a), Figure 12(a), and Figure 13(a) can we see that the inversion curve can better fit the measured curve, and from Figure 10(b), Figure 11(b) ,Figure 12(b), and Figure 13(b). It can be seen that when the inversion algorithm iterative to 400 , the iterative error is less than $1 \mathrm{e}-22$ power, the algorithm can quickly stable convergence.

$\mathrm{L}, . \mathrm{R}, \rho$ are vector in the following four tables ,means that $L=\left[\begin{array}{lll}L 1 & L 2 L 3 L 4\end{array}\right], R=[R]$ $R 2 R 3 R 4], \rho_{=[} \rho_{1} \rho_{2} \rho_{3} \rho_{4}$ ], corresponding to parameter of four segment of pile. The torsion wave velocity $C_{t}$ will be much complicate and the average velocity $C_{t}$ ' can be used to do the error calculating.

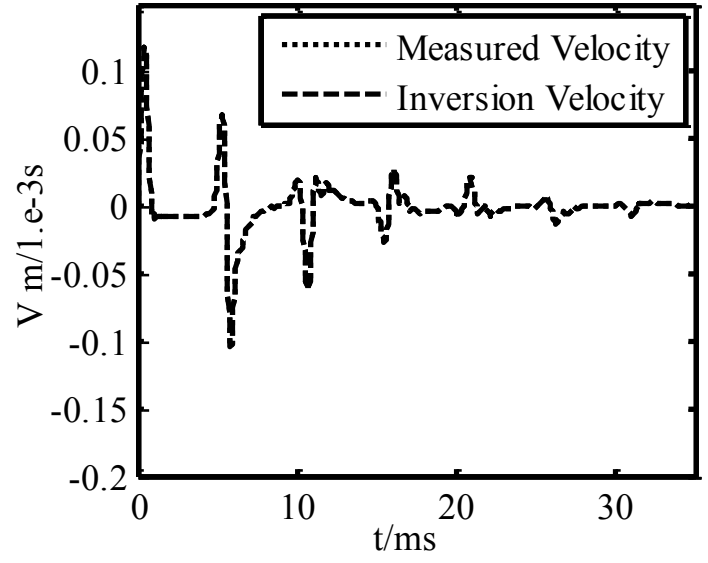

(a)Inversion curve: Velocity - Time

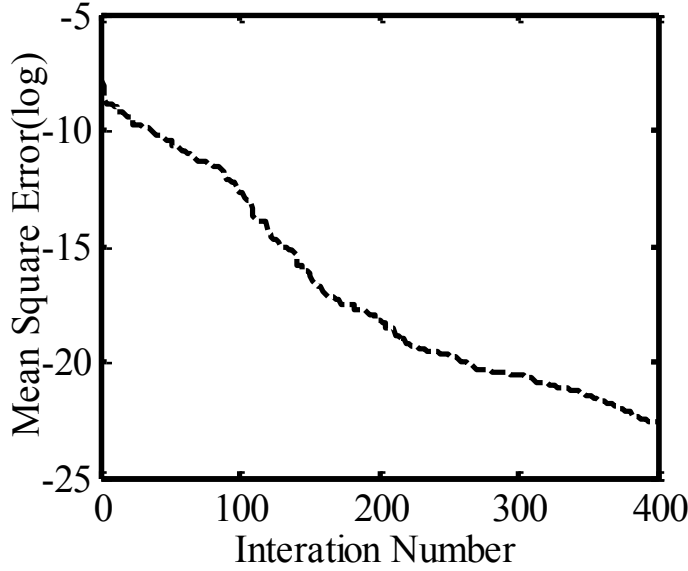

(b) Iterative curve: MSE -Iterations Number

Figure 10. Double Defect -Inversion Analysis for Hole Shrinkage and Hole Enlargement, L1 = 5m; L2 = 5.5m; L3 = 10m; L4 = 20m 
Table 8. Double Defects Iterative Results for Mix of Mud Segregation

\begin{tabular}{c|c|c|c|c}
\hline & \multicolumn{4}{|c}{$\mathrm{L}$} \\
\hline Output & 4.993 & 5.491 & 9.956 & 19.992 \\
\hline Real & 5 & 5.5 & 10 & 20 \\
\hline & \multicolumn{5}{|c}{$\mathrm{R}$} \\
\hline Output & 0.600 & 0.600 & 0.607 & 0.605 \\
\hline Real & 0.6 & 0.6 & 0.6 & 0.6 \\
\hline \multicolumn{5}{|c}{$\rho$} \\
\hline Output & 2397.595 & 2085.834 & 1435.080 & 2397.966 \\
\hline Real & 2400 & 2000 & 1440 & 2400 \\
\hline
\end{tabular}
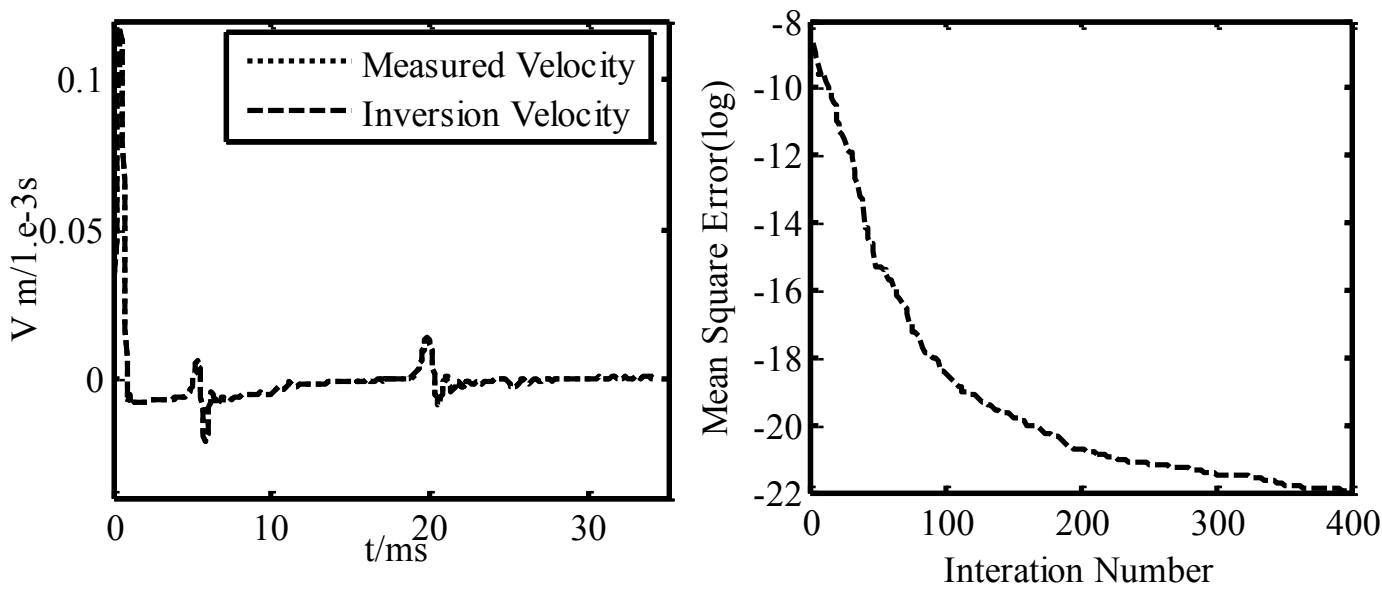

(a)Inversion curve: Velocity - Time (b) Iterative curve: MSE -Iterations Number

Figure 11. Double Defect - Inversion Analysis for Segregation and Mix of Mud, , L1 = 5m; L2 = 5.5m; L3 = 10m; L4 = 20m

Table 9. Double Defects Iterative Results for Hole Shrinkage and Hole Enlargement

\begin{tabular}{c|c|c|c|c}
\hline & \multicolumn{4}{|c}{$\mathrm{L}$} \\
\hline Output & 4.999 & 5.500 & 9.996 & 19.992 \\
\hline Real & 5 & 5.5 & 10 & 20 \\
\hline & \multicolumn{5}{|c}{$\mathrm{R}$} \\
\hline Output & 0.600 & 0.799 & 0.406 & 0.407 \\
\hline Real & 0.6 & 0.8 & 0.4 & 0.4 \\
\hline & \multicolumn{5}{|c}{$\rho$} \\
\hline Output & 2398.425 & 2407.902 & 2363.817 & 2414.528 \\
\hline Real & 2400 & 2400 & 2400 & 2400 \\
\hline
\end{tabular}




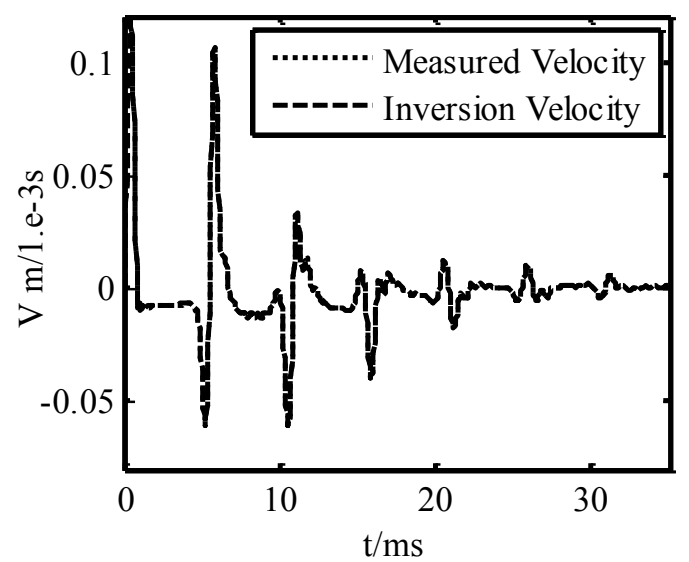

(a)Inversion curve: Velocity - Time

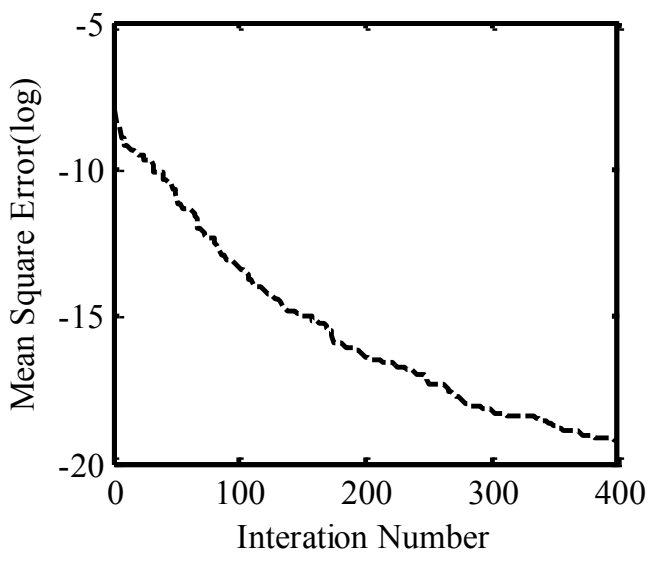

(b) Iterative curve: MSE -Iterations Number

Figure 12. Inversion Analysis for Hole Enlargement and Hole Shrinkage, , L1 $=5 \mathrm{~m} ; \mathrm{L} 2=5.5 \mathrm{~m} ; \mathrm{L} 3=10 \mathrm{~m} ; \mathrm{L} 4=20 \mathrm{~m}$

Table 10. Double Defects Iterative Results for Segregation and Mix of Mud

\begin{tabular}{c|c|c|c|c}
\hline & \multicolumn{5}{|c}{$\mathrm{L}$} \\
\hline Output & 4.995 & 5.499 & 9.995 & 19.992 \\
\hline Real & 5.0 & 5.5 & 10 & 20 \\
\hline & \multicolumn{5}{|c}{$\mathrm{R}$} \\
\hline Output & 0.600 & 0.602 & 0.603 & 0.603 \\
\hline Real & 0.6 & 0.6 & 0.6 & 0.6 \\
\hline \multicolumn{5}{|c}{$\rho$} \\
\hline Output & 2396.775 & 1423.507 & 2048.845 & 2396.693 \\
\hline Real & 2400 & 1440 & 2000 & 2400 \\
\hline
\end{tabular}
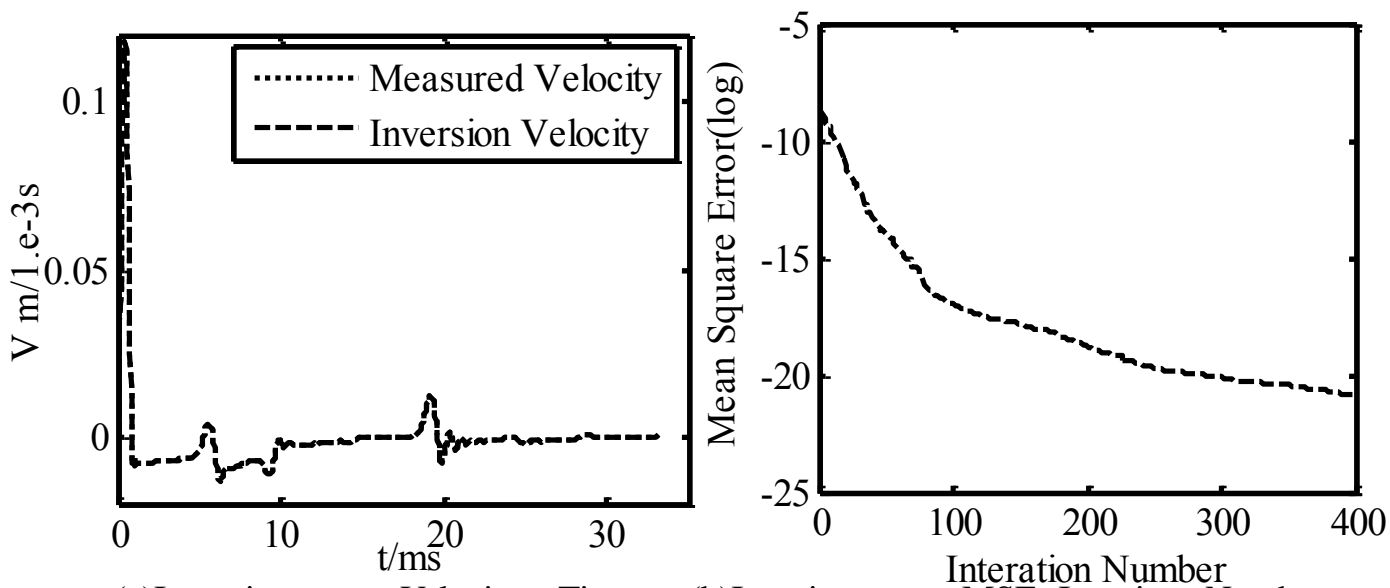

(a)Inversion curve: Velocity - Time

(b)Iterative curve: MSE -Iterations Number

Figure 13. Inversion Analysis for Mix of Mud and Segregation, L1 = 5m; L2 = $5.5 \mathrm{~m} ; \mathrm{L} 3=10 \mathrm{~m} ; \mathrm{L} 4=20 \mathrm{~m}$ 


\section{Table 11. Double Defects Iterative Results for Hole Shrinkage and Hole Enlargement}

\begin{tabular}{c|c|c|c|c}
\hline & \multicolumn{4}{|c}{$\mathrm{L}$} \\
\hline Output & 4.994 & 5.495 & 9.996 & 19.997 \\
\hline Real & 5.0 & 5.5 & 10 & 20 \\
\hline & \multicolumn{5}{|c}{$\mathrm{R}$} \\
\hline Output & 0.600 & 0.400 & 0.804 & 0.797 \\
\hline Real & 0.6 & 0.4 & 0.8 & 0.8 \\
\hline & \multicolumn{5}{|c}{$\rho$} \\
\hline Output & 2400.158 & 2401.851 & 2271.645 & 2458.985 \\
\hline Real & 2400 & 2400 & 2400 & 2400 \\
\hline
\end{tabular}

The calculated average torsion velocity $C_{t}{ }^{\prime}$ are $2011.98,2000.54,2049.16,1998.74$, relative to the real average torsion velocity $2049.8,2000,2016.40,2000$ respectively, the corresponding error of average torsion velocity are $0.32 \%, 0.1 \%, 0.14 \%, 0.26 \%$ respectively.

Combined with Table 8, Table 9, Table 10, Table11 and the output results of parameter iteration by the inversion algorithm, can be seen that the inversion algorithm can accurately mining torsion wave response curves of the related parameters of the pile body, and has high fitting precision.

\section{Conclusion}

In pile foundation dynamic testing Engineering, the inversion analysis of the response of pile top is a very important part. The wave propagation model contains the defect information existed in pile body. And these information should be dig out by inversion algorithm. Traditional inversion algorithm such as the simplex method has weak convergence and robust and its inversion result may confuse the actual situation.

So in this paper, considering the effect of pile soil and subsoil, by combining hybrid artificial bee colony algorithm and finite difference algorithm, a new inversion analysis search algorithm is proposed. The new inversion algorithm was applied to torsion wave response, and three groups experiment had been done for pile foundation under different defect assumption. From the tables and figures discussion above we can draw conclusion that the inversion algorithm can output the pile parameters correctly, the related parameters such as torsion wave velocity, stiff coefficient, damping coefficient can be calculated beyond the error of 3\%, the proposed inversion algorithm has high precision, stable convergence, a reliable qualitative and quantitative analysis of pile defects can be done according to these output parameters..

\section{Acknowledgments}

The authors would like to thank the anonymous reviewers and editors for their valuable comments to improve the presentation of the paper. This work is supported by National Natural Science Foundation of China (NSFC), project number is 61371174.

\section{References}

[1] C. Zheng, H. Liu, X. Ding, and Y. Lv, "Torsional dynamic response of a large - diameter pipe pile in viscoelastic saturated soil," International Journal for Numerical and Analytical Methods in Geomechanics, vol. 38, ( 2014),pp. 1724-1743.

[2] W. Wu, H. Liu, M. H. El Naggar, G. Mei, and G. Jiang, "Torsional dynamic response of a pile embedded in layered soil based on the fictitious soil pile model," Computers and Geotechnics, vol. 80, (2016), pp. 190-198.

[3] Y.-q. Cai, P. Wang, H.-L. Sun, and C.-j. Xu, "Torsional response of a rigid circular foundation on a 
saturated half-space to SH waves," Soil Dynamics and Earthquake Engineering, vol. 30, (2010),pp. 1082-1088.

[4] S. Basack and S. Sen, "Numerical solution of single piles subjected to pure torsion," Journal of Geotechnical and Geoenvironmental Engineering, vol. 140, (2013),pp. 74-90.

[5] X. Ding, H. Liu, G. Kong, and C. Zheng, "Time-domain analysis of velocity waves in a pipe pile due to a transient point load," Computers and Geotechnics, vol. 58, (2014),pp. 101-116.

[6] W. Jinhai, "Inversion of 3-parameter theory of laterally-loaded pile," Journal of Jiangsu University of Science and Technology (Natural Science Edition), vol. 1,( 2011), p. 004.

[7] K.-1. JIANG, Y.-p. LI, R.-m. ZHANG, and Q.-w. ZHANG, "Inversion analysis of soil resistance factor of plastic concrete cut-off wall," Rock and Soil Mechanics, vol. 33, ( 2012),pp. 389-395.

[8] J.-g. Chen, T.-q. Zhou, R.-c. Hu, and H.-y. Tian, "A Genetic Algorithm for parameter Inversion of Integrated Pile in Dynamic Testing,"Rock and Soil Mechnics,vol.45,(2015),pp.125-145.

[9] D.-j. Liu, "Frequency-domain solution of shear stress along pile-soil interface for longitudinal vibration of piles and its application," CHINESE JOURNAL OF GEOTECHNICAL ENGINEERING-CHINESE EDITION-, vol. 23,( 2001), pp. 544-546.

[10] G. Militano and R. Rajapakse, "Dynamic response of a pile in a multi-layered soil to transient torsional and axial loading," Geotechnique, vol. 49, (1999),pp. 91-109.

[11] D. Karaboga, "An idea based on honey bee swarm for numerical optimization," Technical report-tr06, Erciyes university, engineering faculty, computer engineering department,( 2005),pp.120-136.

[12] Z.-b. KE, D.-j. LIU, and X.-t. LIAO, "Numerical calculation of torsional vibration of intact piles," Journal of Hefei University of Technology (Natural Science), vol. 11,( 2005), p. 021,.

[13] S. Abd-Elazim and E. Ali, "A hybrid particle swarm optimization and bacterial foraging for optimal power system stabilizers design," International Journal of Electrical Power \& Energy Systems, vol. 46, (2013),pp. 334-341.

[14] K. S. Kumar and T. Jayabarathi, "Power system reconfiguration and loss minimization for an distribution systems using bacterial foraging optimization algorithm," International Journal of Electrical Power \& Energy Systems, vol. 36,( 2012), pp. 13-17.

[15] J. Luo and Y. Li, "Artificial bee colony algorithm with chaotic-search strategy," Control and Decision, vol. 25, (2010),pp. 1913-1916.

\section{Authors}
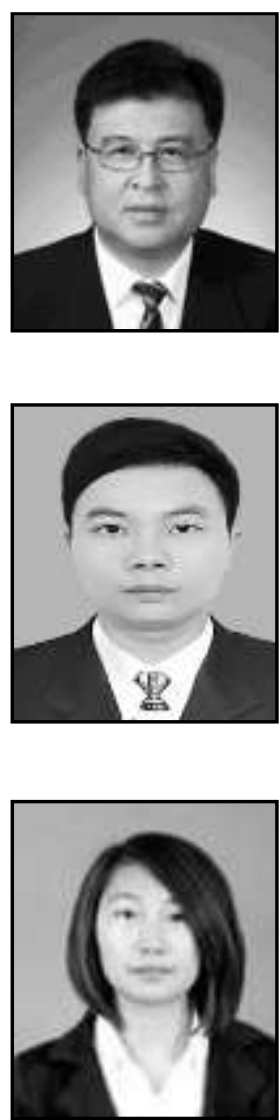

Kang Weixin, he was born in Heilongiiang province, China in 1963. He received his Ph.D. degree in instrument science and technology from Harbin Institute of Technology, China in 2010.Now he is a professor in the College of Information And Communication Engineering, Harbin Engineering University, Harbin, China. His current research interests include modern digital communication system and communication technology and information intelligent detection theory.

Ye Youdao received his bachelor B.Sc. degree from Harbin engineering university in 2014.Currently in information and communication engineering major graduate, Harbin engineering university, his main research direction is swarm intelligence algorithm, the computer simulation, signal detection.

Zhao Tianwei, received her bachelor B.Sc. degree in 2012 from Heilongjiang Institute of Science and Technology, now she is a master student in Harbin Engineering University. Her main research interests is Signal detection and processing. 
International Journal of Signal Processing, Image Processing and Pattern Recognition Vol. 10, No. 8 (2017) 